\title{
Performance of Implementation IBR-DTN and Batman-Adv Routing Protocol in Wireless Mesh Networks
}

\section{Herman Yuliandoko, Sritrusta Sukaridhoto, M Udin Harun Al Rasyid, Nobuo Funabiki}

\author{
Graduate of Information Engineering and Computer, Electronics Engineering \\ Polytechnic Institute of Surabaya \\ Graduate School of Natural Science and Technology, Okayama University, Japan \\ Herman.Yuliandoko@yahoo.com; dhoto@pens.ac.id; udinharun@pens.ac.id; \\ funabiki@cne.okayama-u.ac.jp
}

\begin{abstract}
Wireless mesh networks is a network which has high mobility and flexibility network. In Wireless mesh networks nodes are free to move and able to automatically build a network connection with other nodes. High mobility, heterogeneous condition and intermittent network connectivity cause data packets drop during wireless communication and it becomes a problem in the wireless mesh networks. This condition can happen because wireless mesh networks use connectionless networking type such as IP protocol which it is not tolerant to delay. To solve this condition it is needed a technology to keep data packets when the network is disconnect. Delay tolerant technology is a technology that provides store and forward mechanism and it can prevent packet data dropping during communication. In our research, we proposed a test bed wireless mesh networks implementation by using proactive routing protocol and combining with delay tolerant technology. We used Batman-adv routing protocol and IBR-DTN on our research. We measured some particular performance aspect of networking such as packet loss, delay, and throughput of the network. We identified that delay tolerant could keep packet data from dropping better than current wireless mesh networks in the intermittent network condition. We also proved that IBR-DTN and Batman-adv could run together on the wireless mesh networks. In The experiment throughput test result of IBR-DTN was higher than Current TCP on the LoS (Line of Side) and on environment with obstacle.
\end{abstract}

Keywords: Delay Tolerant, IBR-DTN, Wireless Mesh, Batman-adv, Performance

\section{INTRODUCTION}

Wireless mesh networks is a computer network which each nodes or users connected and relay data to the network. In the mesh network each node operates not only as a host but also as a router [1]. Compared with 
conventional wireless network, this wireless network is very flexible, more efficient energy to cover large coverage area by using multi hop nodes. In the wireless mesh network also have routing protocol to determine route from transmitter node to receiver node. This protocol is needed because the transmission data from transmitter to receiver node will trough some intermediate nodes and to get best route. There are several considerations such as bandwidth and range to choose best route. Wireless mesh networks have two model routing protocol, reactive routing protocol and proactive routing protocol. The implementation of wireless mesh networks with a routing protocol can improve performance of network but the data packets are vulnerable to drop. This condition can be happen when networks in the intermittent connection.

To solve communication problem we use delay tolerant network technology. Delay Tolerant Network (DTN) is an approach to computer network architecture that seeks to address the transmit data problem on intermittent network connectivity by store and forward mechanism. In the implementation of wireless mesh network we use Batman-adv to provide routing protocol algorithm. We combined this wireless mesh network with IBR-DTN as store and forward tool mechanism.

Research on DTN implementation brings benefits in many areas although research in this area is still rarely done. In the fields of medical, DTN applications was used to support long delay and distance communication by using VoIP with DTN application to connect doctor and patients in the remote areas [2]. DTN also was used in data transmission on Vehicular Network in Intelligent Transport System [3]. In our previous research we made a design implementation of DTN on the urban area [4] and in this research we implemented DTN on wireless mesh networks to improve the ability wireless mesh network on intermittent communications.

Batman-adv is one of mesh routing protocol which use proactive routing protocol mechanism and uses distance vector mechanism to determine the best route. The distance vector is very useful for mesh architecture because it is easy to change the route of nodes connection. That why Batman-adv is better running on high mobility [5]. Batman-adv also can run in the two layer protocol, layer 2 and layer 3. In the layer 3 like most other routing protocol and Batman-adv routing protocol on layer 2 is introduced for better performance [6]. Batman-adv protocol use Transmission Quality (TQ) metric to find a stable link among another node. In Batman-adv also have mechanism in the all of nodes to send OGM (Originator Messages) to inform another nodes about their existence. This OGM transmitted in the fix intervals to the around nodes and it will retransmit to another nodes. This routing protocol was combined with IBRDTN as DTN tool. IBR-DTN is one of DTN tool which prove more efficient [7] compare to another DTN tools.

The remainder of this paper is organized as follows: in Section II we discuss related research on DTN and routing protocol of wireless mesh 
networks. The originality of this research in Section III, focusing on improvement wireless mesh networks through additional IBR-DTN mechanism and Batman-adv routing protocol. Section IV described about design system including measurement method to check performance of wireless mesh networks. Experiment design on Section V contains test bed scenario, result of examination, and analysis. In the end of this paper is conclusion and future work.

\section{RELATED WORKS}

Study on wireless mesh implementation had been done by Kharisma Babu et al. [1]. That implementation was focused on two routing protocol model, OLSR and Batman. It was also studied the performance of different topologies using static routing protocol and the result showing that Batman was the most efficient protocol for mesh networks.

Davinder et al. [5] had evaluated the performance of Batman, DSR, OLSR routing protocol. The evaluation of this routing protocol was based of packet delivery ratio, end to end delay, routing load and throughput measurement. The evaluation by using simulation with number of node was 100 nodes and Batman routing protocol showed better result when mobility was high.

Daniel Seiter et al. [6] made a practical approach to investigate BatmanAdv routing protocol performance. The performance of Batman-Adv routing protocol evaluation was done in real world office environment to know main parameters influencing the routing performance such radio conditions, delay, and throughput and packet loss. The performance evaluation was proven to work as well in an environment with significant interference.

One of DTN tools is IBR-DTN and IBR-DTN was showing as an efficient tool to implement for embedded system. The practical evaluation had done by Michael Doering on his research [7]. The research showed that IBR-DTN not only slim application for embedded system but also suitable to running on embedded system due to lower consumption of memory.

Communication in disaster area networks is vital and the quality of communication is very unpredictable and unstable. It needs communication mechanism to cover it. Larent Delosieres et al. [8] had make simulation by using NS3 to add store-and-forward mechanism to the routing component in Batman nodes. The store-and-forward mechanism is mechanism to reduce the risk of losing data due to intermittent connection.

\section{ORIGINALITY}

This research proposes to implement delay tolerant network on wireless mesh networks. In this research we also use Batman-adv routing protocol which have flexibility on the high mobility and we add wireless mesh networks with IBR-DTN as delay tolerant networks tool. This IBR-DTN take advantages to the wireless mesh networks with store and forward mechanism. This mechanism prevents data loss on the intermittent network 
condition. Research analysis in our experiment was based on real world condition test bed. The best my knowledge this is first time the combination of Batman-adv and IBR-DTN on real condition test bed. In our research we did deeply analysis of throughput, delay time and packet loss on the real world condition. This analysis can be a good contribution to the next research of wireless mesh network implementation. Besides that the resulting formula of experiment can facilitate further research in predicting the value of delay time and throughput. Wireless mesh with high flexible mobility including store and forward mechanism is needed in unpredictable environment condition now.

\section{SYSTEM DESIGN}

In wireless mesh networks each nodes are self-organized wireless networks, and operates not only as a host but also a router. Compared to a conventional wireless networks, a wireless mesh networks can achieve the same coverage area with lower transmission power through multi-hop communication [1].

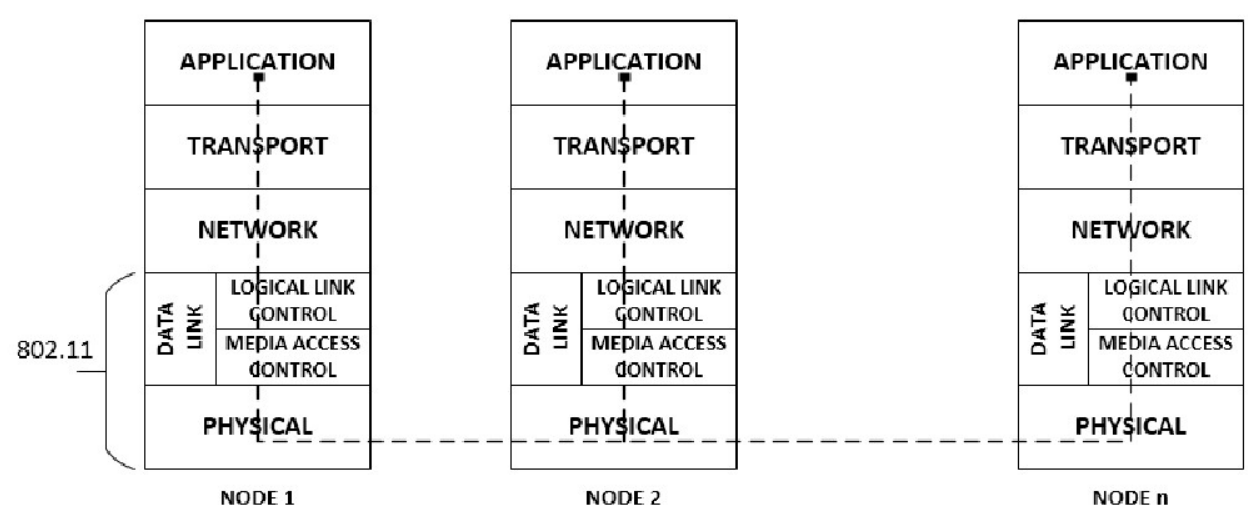

Figure 1. Current Wireless Mesh Layer [10]

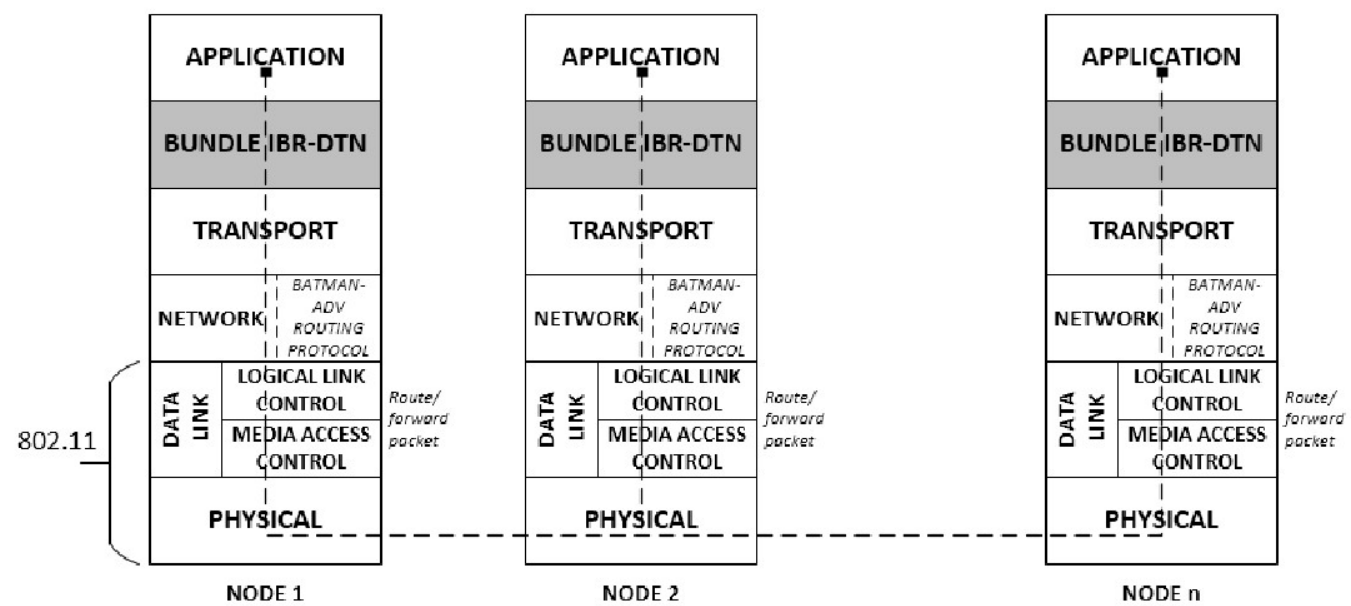

Figure 2. Wireless Mesh Layer with IBR-DTN and Batman-adv 


\subsection{Batman-adv Routing Protocol}

Better Approach to Mobile Ad Hoc Network Advance (Batman-adv) is a proactive routing protocol with distance vector mechanism. Every node on Batman-adv transmits OGM to another node in fix duration and the approach of Batman-adv algorithm is to divide the knowledge about the best end-toend paths between nodes in a mesh network. Each node perceives and maintains only the information about the best next hope.

The algorithm of Batman-adv can be described as follows. Each node in mesh network transmits Originator Messages (OGM) to inform about the existence of node. This message from originator of OGM retransmit to the another neighbor. It make the network is flooded with originator messages. OGM transmit in the fix periodic and contain a small packet size 52 byte include IP and UDP overhead. The flooding mechanism make routing information same and prevent routing loop. Batman-adv also can work in the layer $3^{\text {th }}$ like OLSR. It means Batman-adv use IP address to communicate with another node. Although Batman-adv only pays attention to the best next hop, it makes Batman-adv more efficient and faster.

Batman-adv already developed for open source software and www.open-mesh.org become main development website. We can download and use Batman-adv software from this website. Batman-adv also can be implemented in the various operation system and we use Ubuntu 14.04 LTS (Trusty Tahr) in our research. To implement Batman-adv in Ubuntu we should prepare some libraries and software to support it.

Step 1: Prepare libraries, Batctl, and Batman-adv

Step 2: Create mode ad-hoc, ssid and channel

Before we create an ad-hoc network, it is needed to disable our Ubuntu firewall and line down wireless network interface (wlan0).

\#iwconfig wlan0 mode ad-hoc essid herman channel 1

In the mesh network must have same essid and channel in the all of nodes.

Step 3 : Batman activation

Because of Batman-adv also can run on $2^{\text {nd }}$ network layer, Batman-adv supported by batctl. Batctl is the configuration and debugging tool for Batman-adv.

\#./batctl if add wlano

\#ifconfig wlan0 mtu 1527

\#cat/sys/class/net/wlan0/batman_adv/iface_status

\#ifconfig wlano [ip address]

\#ifconfig bato [ip address] up. 


\subsection{IBR-DTN}

DTN overcome the problems associated with intermittent connectivity, long or variable delay, asymmetric data rates, and high error rates by using store-and-forward message switching. This is an old method, used by ponyexpress and postal systems since ancient times.

IBR-DTN is a fully RFC5050 [8] compliant implementation of Bundle Protocol. The Linux System becomes main focus of application of IBR-DTN especially on the embedded system. The web site on www.ibr.cs.tubs.de/projects/ibr-dtn/ is main development web site focus in IBR-DTN.

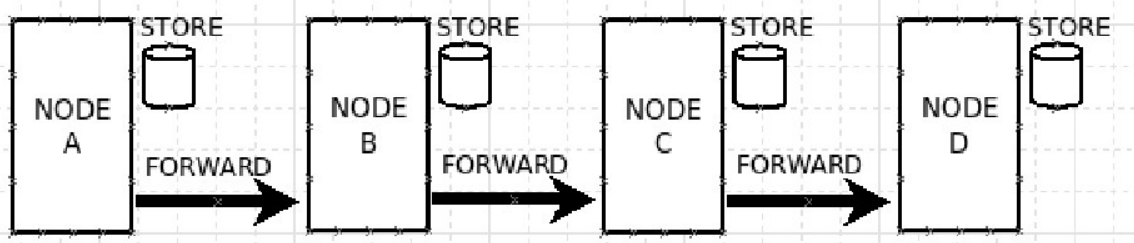

Figure 3. Store and Forward [9]

To implement IBR-DTN we can use repository of IBR-DTN or use manual configuration of IBR-DTN Daemon and Tools

- ibrcommon-0.12.1.tar.gz

- ibrdtn-0.12.1.tar.gz

- ibrdtnd-0.12.1.tar.gz

- ibrdtn-tools-0.12.1.tar.gz.

\subsection{Measurement and Method}

A perfect network should be able to provide a route between node to another node in the network and the packets that transmitted from node to another node without being dropped is priority of data transmission. Delay transmission and throughput also become a priority to know the performance of networking as below formula [8].

a. Packet Delivery Ratio (PDR)

It is a comparison between packet transmitted by sender (Ps) and packet received (Pr).

$$
\begin{gathered}
P D R=\left(\frac{P r}{P_{s}}\right) \times \mathbf{1 0 0} \\
\operatorname{Pr}=\text { packet } \text { received } \\
\text { Ps }=\text { packet transmitted } / \text { sent }
\end{gathered}
$$

b. End to end delay (Davg)

Davg is delay transmitting compare to packet received. It is needed to know because packet size also influence to the performance of communication.

$$
\operatorname{Davg}=\sum(\mathrm{tr}-\mathrm{ts}) / \mathrm{Pr}
$$




$$
\begin{aligned}
& \operatorname{Pr}=\text { packet received } \\
& \operatorname{tr}=\text { packet receive time } \\
& \mathrm{ts}=\text { packet send time }
\end{aligned}
$$

Tiphon (Telecommunication and Internet Protocol Harmonization Over Networks) mention on the General aspects of Quality of Service, that classification of delay time as below table:

Table 1. Delay Category [11]

\begin{tabular}{|c|c|c|}
\hline Category & Delay (ms) & Index \\
\hline Very good & $<150$ & 4 \\
\hline Good & $150-300$ & 3 \\
\hline Fair & $300-450$ & 2 \\
\hline Poor & $>450$ & 1 \\
\hline
\end{tabular}

c. Throughput, the total successfully received packet to the destination. Throughput is actual bandwidth, it is usually measured in bits per second (bit/s or bps), and sometimes in data packets per second (p/s or pps) or data packets per time slot.

\section{EXPERIMENT}

\subsection{Experiment Design}

Hardware Platform

\begin{tabular}{ll}
\hline Hardware platform & $\begin{array}{l}\text { Asus 452C (Node A), Lenovo S410p (Node B), } \\
\text { Lenovo G450 Dual Core (Node C) }\end{array}$ \\
\hline USB Wifi Dongle & TL-WN722N \\
\hline USB Wifi Chipset & Atheros AR9271 \\
\hline USB Wifi Specification & Atheros AR9271 802.11n \\
Chipset & $2.400-2.4835 \mathrm{GHz}$ \\
Srequency & $11 \mathrm{n}:$ Up to 150 Mbps \\
& $11 \mathrm{~g}:$ Up to 54 Mbps \\
Transmit Power & $11 \mathrm{~b}:$ Up to 11 Mbps \\
Wireless Modes & $<20 \mathrm{dBm}$ \\
Antenna Gain & Ad-hoc / Infrastructure \\
\hline & $4 \mathrm{dBi}$ \\
\hline Software Platform & \\
\hline Operating System & Ubuntu 14.04 LTS \\
\hline Batman-Adv & Batman-adv-2014.0.0 \\
\hline IBR-DTN & IBR-DTN-0.12.1 \\
\hline Wifi Monitoring & Wireshark, LinSSID \\
\hline Remote network & Desktop Sharing, Remmina \\
\hline Throughput monitoring & Iperf \\
\hline Desktop recorder & Kazam \\
\hline
\end{tabular}


We done some experiment to test the performance of IBR-DTN compare to the Current TCP and it done in the two environment condition LOS (Line of Side) and environment with many obstacles. For the environment with many obstacles we chose 2rd floor of PENS D3 Campus Building as below illustration picture.

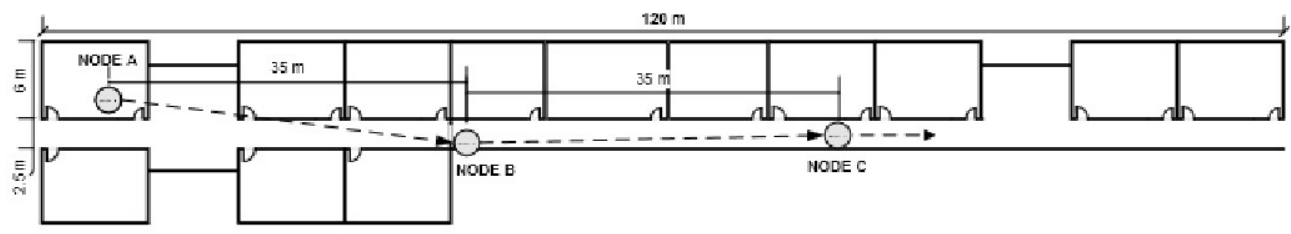

Figure 4. Map of PENS D3 Campus Building 2nd floor

We tested the performance of IBR-DTN and Batman-adv by using ICMP (Internet Control Message Protocol). ICMP is one of protocol on internet networking. It is used by node or network devices to check connection or error message indicating. Before we did performance test by using ICMP test make sure that all nodes already on the ad-hoc mode and batman-adv interface on active status.

In this test we used ping and dtnping (for IBR-DTN) to send ICMP between nodes. Ping test was used for network condition without delay tolerant application. For this test we send data in the same size with below command:

\#ping -s [packet size] -c [count] [IP address destination]

To run IBR-DTN on the our network we needed to activate IBR-DTN daemon and combine IBR-DTN with wireless mesh network interface including batman routing protocol

\#/etc/init.d/ibrdtn stop

\#dtnd -i bat0 -c /etc/ibrdtn/ibrdtn.conf

Make sure there was not error on the daemon running interface. We continued the ICMP sending process by

\#dtnping -size [packet size] -count [count] dtn://[user destination]/echo

We also use Iperf to do throughput test. iperf [-s |-c host] [option]

- -s |-c : node as server (-s) or as client (-c)

- option : -i interval, second between periodic bandwidth reports

-p port, server port to listen on/connect to

-n num, number of bytes to transmit (instead of $-\mathrm{t}$ )

- $t$ time, time in seconds to transmit for (default $10 \mathrm{sec}$ ) 
-b bandwidth, for UDP bandwidth to send at in bits/sec (default $1 \mathrm{Mbit} / \mathrm{sec}$, implies $-\mathrm{u}$ )

-u use UDP rather than TCP

-D daemon, run the server as daemon

-V IPv6version, set the domain to IPv6

In this experiment we use

$$
\begin{array}{ll}
\text { \#iperf-s -i } 1 & \text { (server) } \\
\text { \#iperf -c [ip address server] -i 1-n 5G } & \text { (client) }
\end{array}
$$

By using Parson correlation coefficient method we measured the correlation of variable data and node distance to the delay time.

Table 2. Interpretation of Correlation Coefficient [12]

\begin{tabular}{|c|c|}
\hline $\begin{array}{c}\text { Correlation } \\
\text { Coefficient Value }\end{array}$ & $\begin{array}{c}\text { Direction and Strength of } \\
\text { Correlation }\end{array}$ \\
\hline-1 & Perfectly negative \\
\hline-0.8 & Strongly negative \\
\hline-0.5 & Moderately negative \\
\hline-0.2 & Weakly negative \\
\hline 0 & No association \\
\hline+0.2 & Weakly positive \\
\hline+0.5 & Moderately positive \\
\hline+0.8 & Strongly positive \\
\hline+1 & Perfectly positive \\
\hline
\end{tabular}

\subsection{Experiment and Analysis}

\subsubsection{Experiment 1 (Node on fixed position, on obstacle environment)}

This experiment was done in the 2rd floor of PENS D3 Campus Building with all nodes in the fix position.

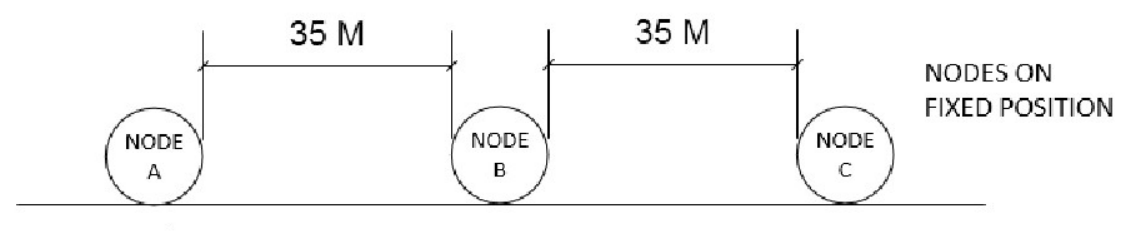

NODE A on the room

Figure 5. Node Position on Experiment 1

Node A sent data to the Node $\mathrm{C}$ trough Node $\mathrm{B}$, and it was done by using dtnping for IBR-DTN mode and ping for Current TCP mode. In this process we also measured throughput by using iperf. 


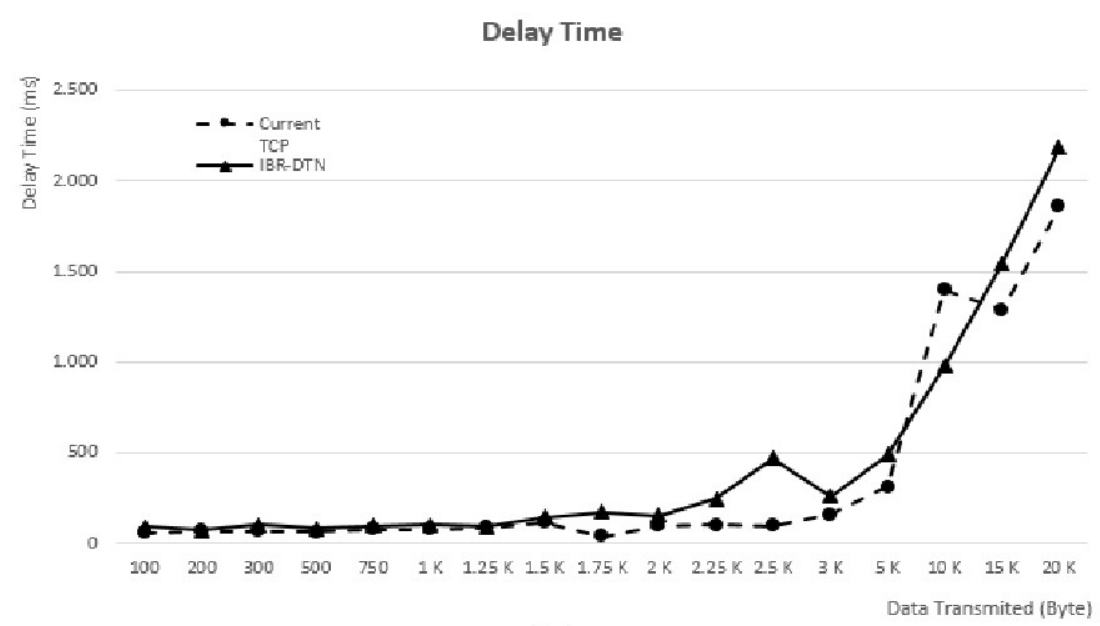

(a)

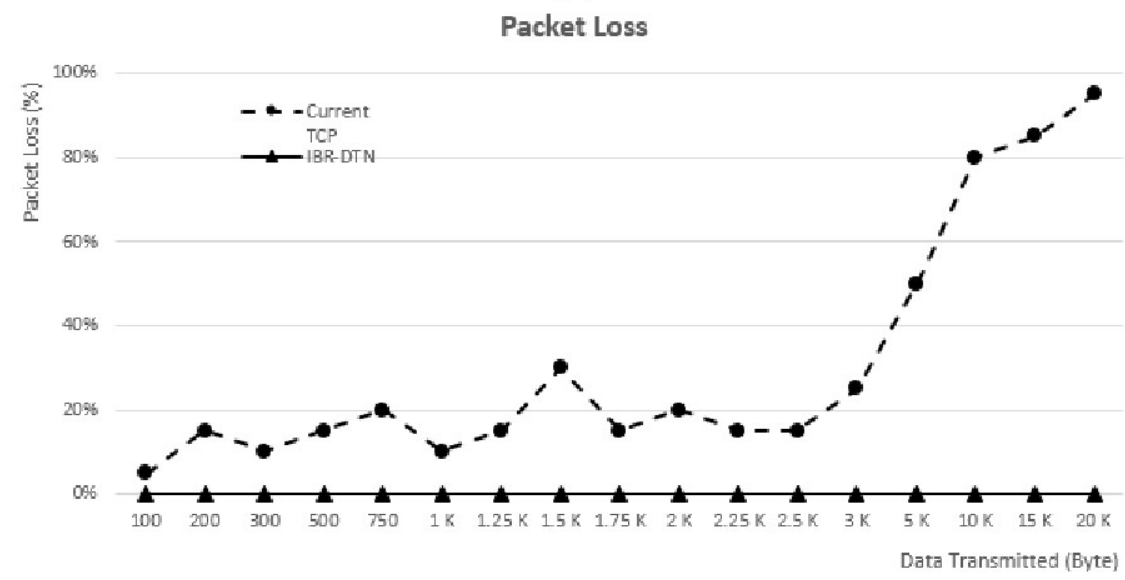

(b)

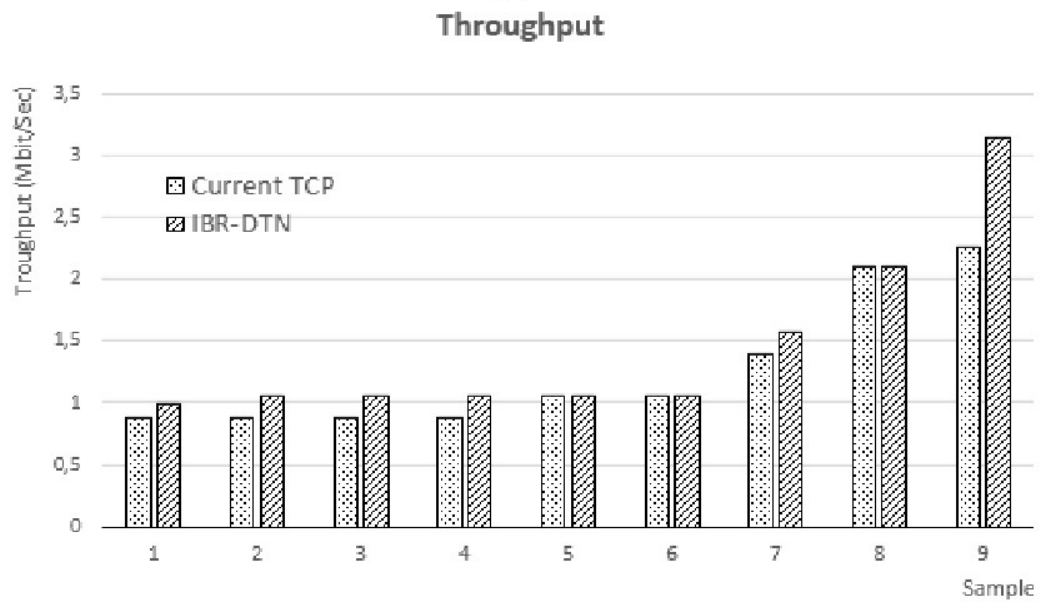

(c)

Figure 6. Delay Time (a), Packet Loss (b) and Throughput (c) (Node on fix position and obstacle environment)

Based on above data, IBR-DTN can work smoothly together with Batman-adv routing protocol. The delay time for data transmitted size more than $5 \mathrm{~K}$ had very poor category in the both of IBR-DTN and Current TCP [11]. By 
implementing IBR-DTN on this experiment could increase throughput result up to $11 \%$ compare to Current TCP. Packet loss in IBR-DTN was better than Current TCP because IBR-DTN had store and forward mechanism and this mechanism increased the packet loss performance up to $31 \%$ better than Current TCP.
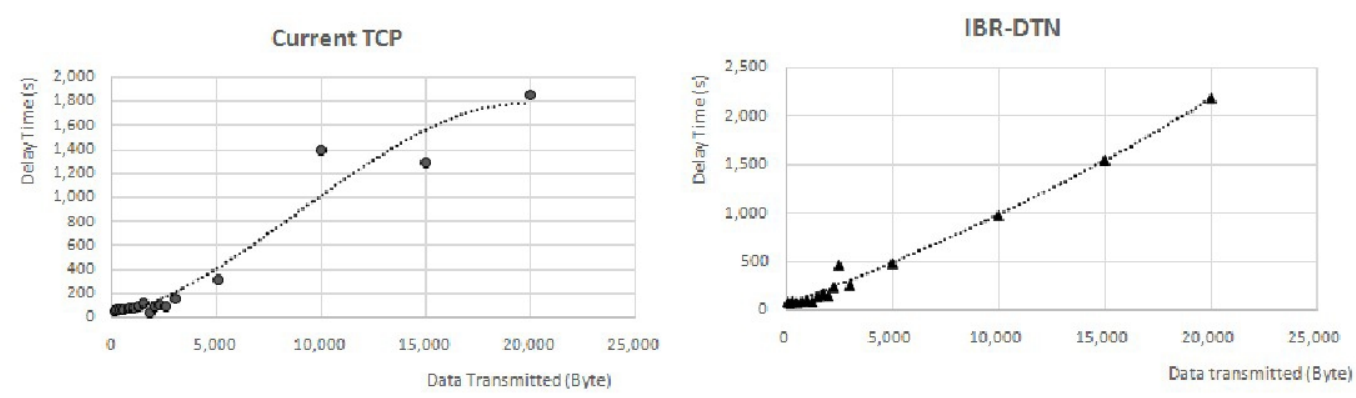

Figure 7. Delay Time to Size of Data Transmitted

(Node on fix position and obstacle environment)

By using statistic tool we also analyzed the delay time trend on the Current TCP and IBR-DTN. This analysis provided delay time polynomial function on the Current TCP and IBR-DTN as below:

- Current TCP

$$
\mathrm{Y}=-7 \cdot 10^{-7} \cdot \mathrm{X}^{2}+0.1105 . \mathrm{X}-51.55
$$

- IBR-DTN

$$
\begin{aligned}
& Y=2 \cdot 10^{-11} \cdot X^{3}+7 \cdot 10^{-7} \cdot X^{2}+0.085 \cdot X-45.205 \\
& \mathrm{Y}=\text { Delay time (s), } \\
& \mathrm{X}=\text { Data Transmitted Size (Byte) }
\end{aligned}
$$

\subsubsection{Experiment 2 (Node on mobile position, on Obstacle environment)}

In this scenario, the position of Node A and B was fixed but Node $\mathrm{C}$ was mobile. Then Node C sent ICMP data to the Node A on mobile position with packet data size was 2000 bytes to test delay time and packet loss. The throughput test by using iperf software.

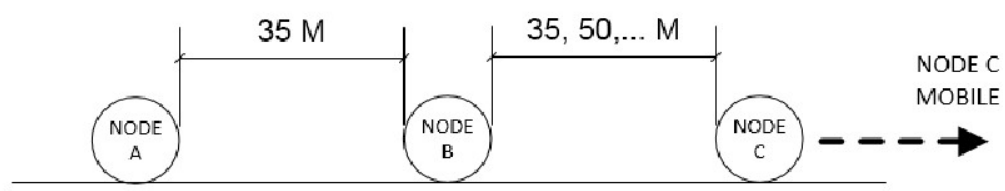

NODE $A$ on the room

Figure 8. Node Position on Experiment 2 


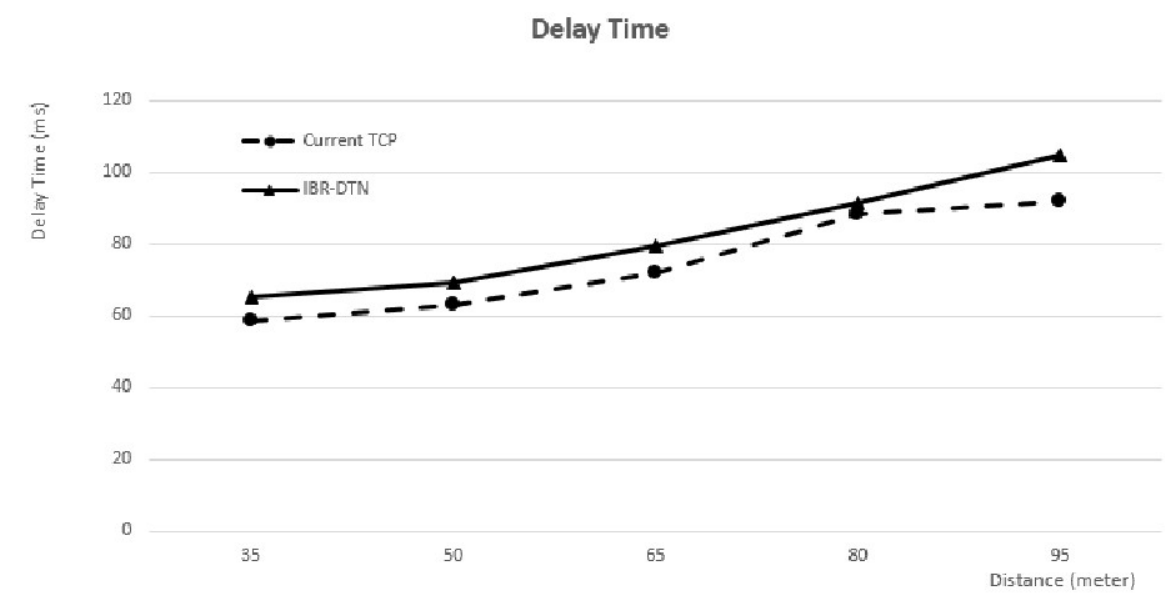

(a)

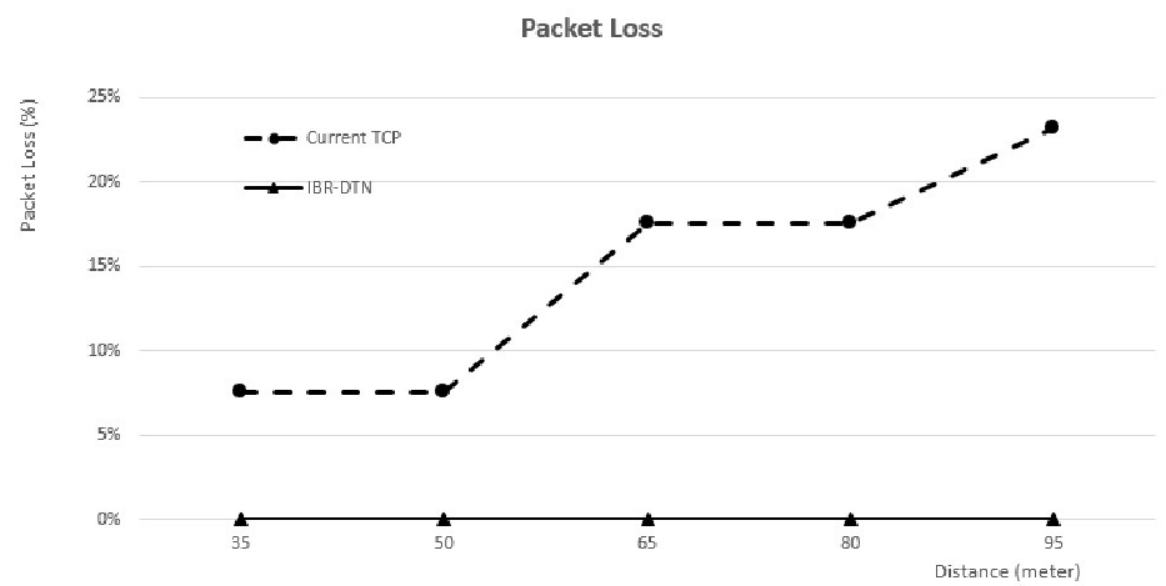

(b)

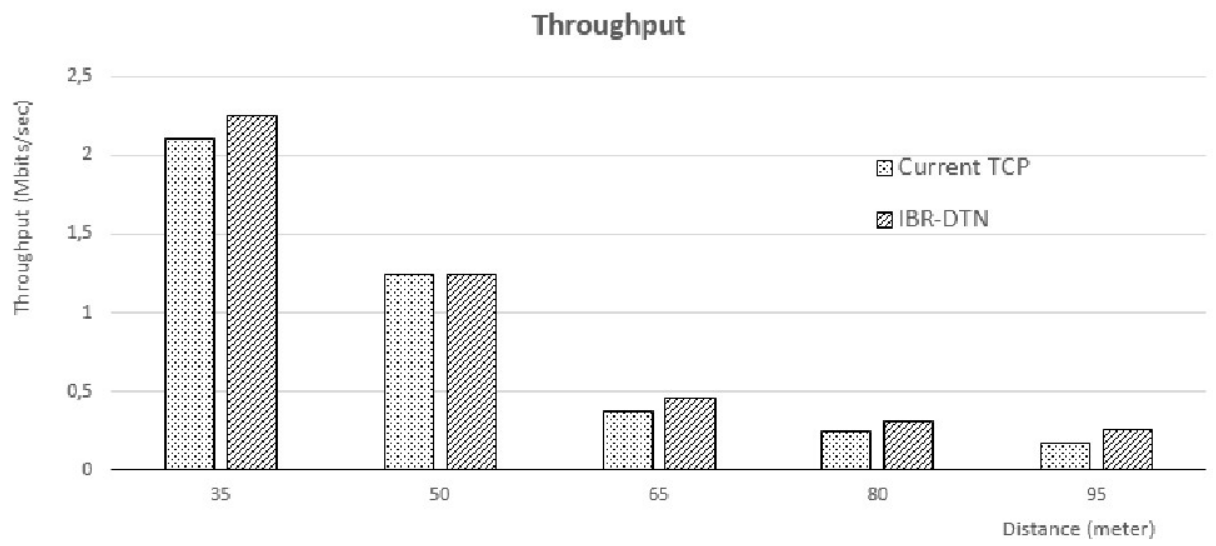

(c)

Figure 9. Delay Time (a), Packet Loss (b) and Throughput (c) (Node $\mathrm{C}$ on mobile position and obstacle environment)

According to above graph we can see that IBR-DTN able to prevent packet data loss during wireless mesh communication and the throughput result also $16 \%$ better than Current TCP. 

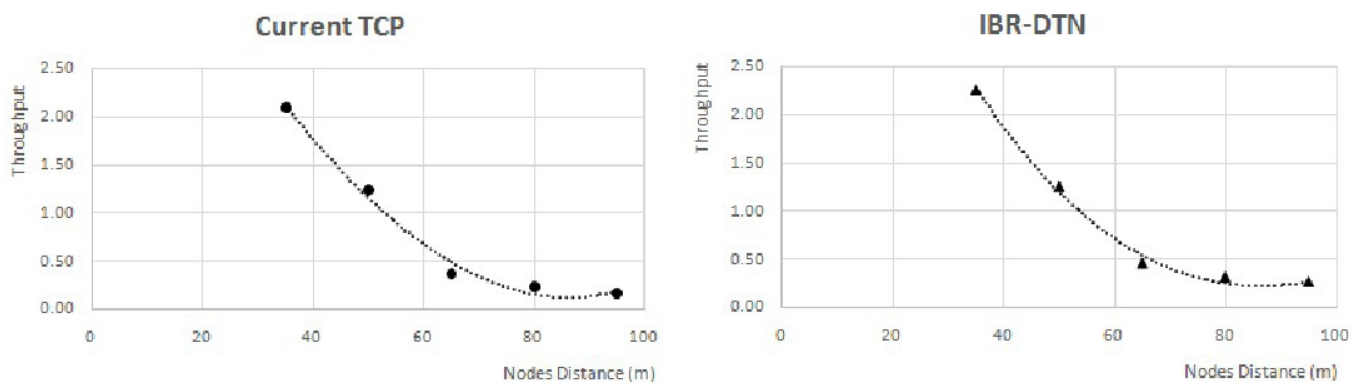

Figure 10. Throughput to Nodes Distance

(Node $\mathrm{C}$ on mobile position and obstacle environment)

We also obtained the corelation between throughput with nodes distance by provided polynomial function.

- Current TCP

$$
\mathrm{Y}=2.10^{-6} \cdot \mathrm{X}^{3}+0.0004 \cdot \mathrm{X}^{2}-0.1062 . \mathrm{X}+5.292
$$

- IBR-DTN

$$
\mathrm{Y}=-3 \cdot 10^{-6} \cdot \mathrm{X}^{3}+0.0014 . \mathrm{X}^{2}-0.1757 . \mathrm{X}+6.842
$$

Formula 5 and 6 indicate the corelation of throughput with nodes distance. Y is throughput and $\mathrm{X}$ is nodes distance.

\subsubsection{Experiment 3 (Node on Fixed position, on LoS Condition)}

In this experiment we want to know throughput performance of IBRDTN compare to Current TCP on the LoS (Line of Side) condition with fixed nodes position. We evaluated the throughput, delay and packet loss by using ICMP message and iperf software.

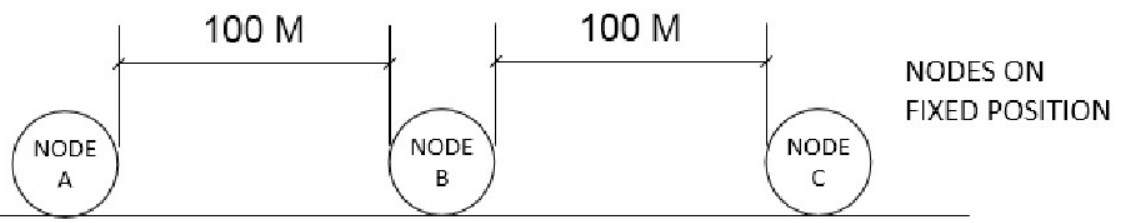

Figure 11. Node Position Experiment 3 


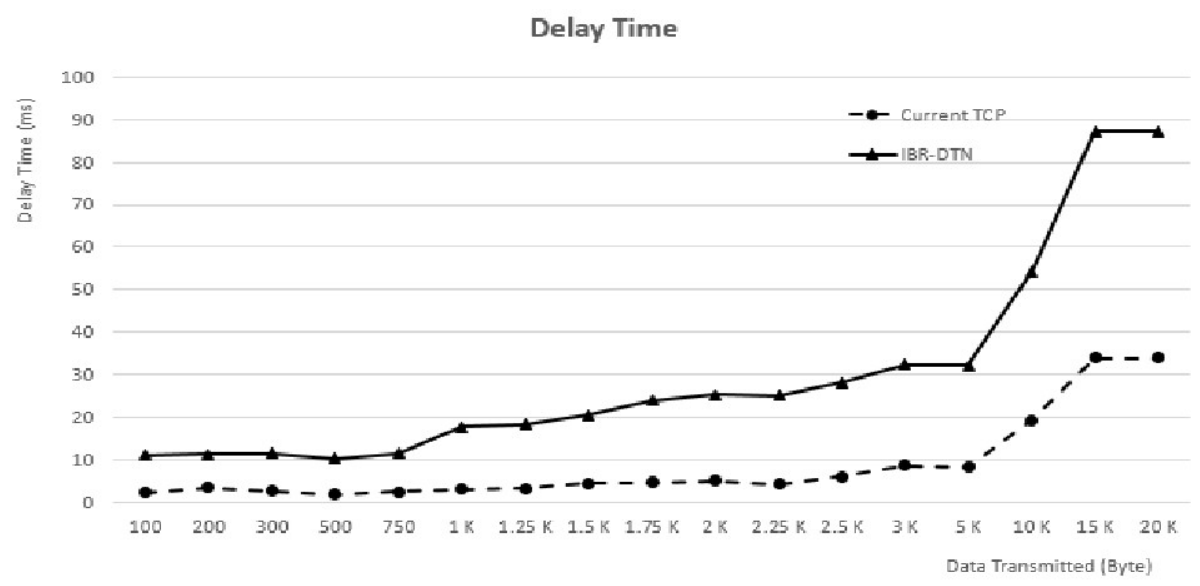

(a)

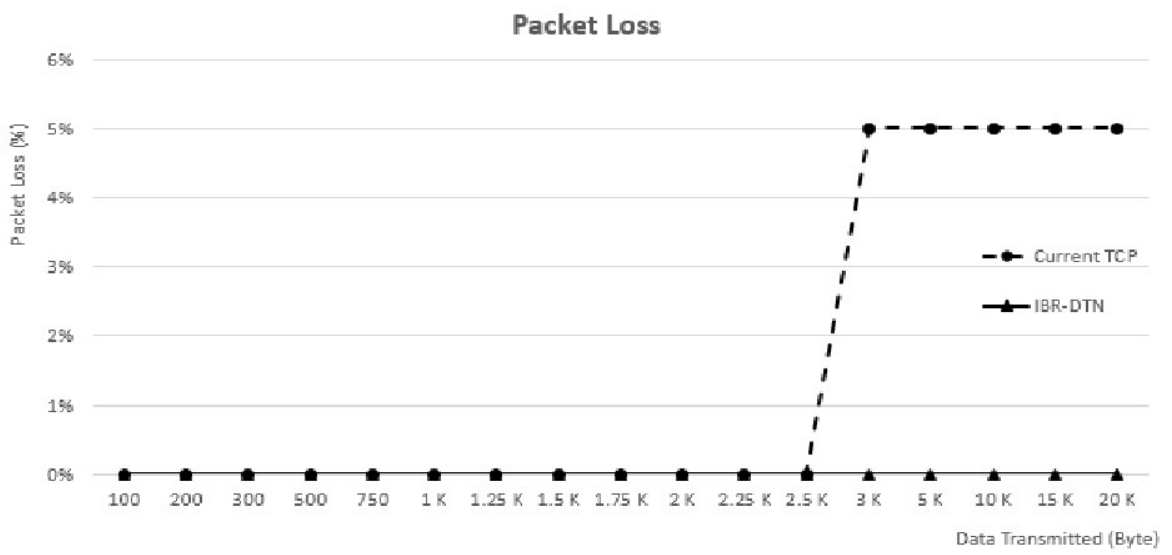

(b)

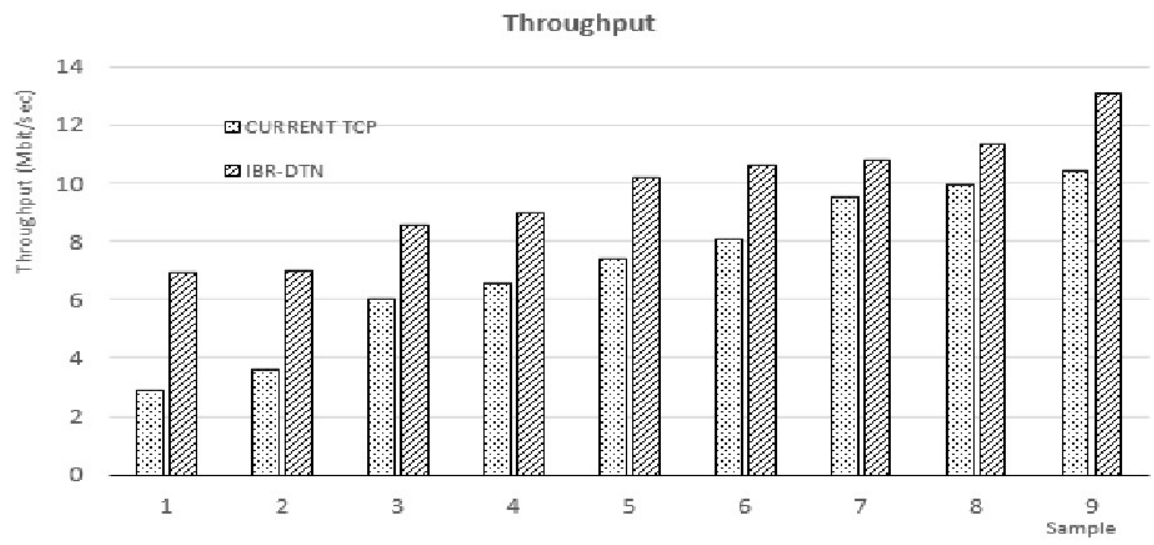

(c)

Figure 12. Delay Time (a), Packet Loss (b) and Throughput (c) (Fix position on LoS condition)

In the LoS environment mode communication, throughput result of IBR-DTN was still better than Current TCP and the average differentiate of throughput was 29\%. The Packet Loss of Current TCP below 2,5K Byte was zero but it 
was began to increase on the $3 \mathrm{~K}$ Byte. In the IBR-DTN by using store and forward mechanism could prevent packet loss.
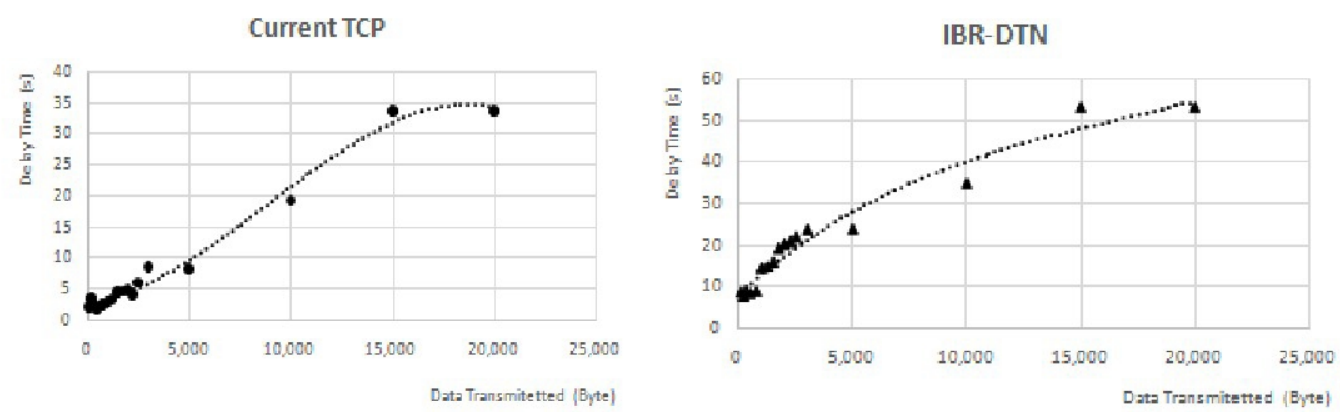

Figure 13. Delay Time to Size of Data Transmitted

(Fix position on LoS condition)

The correlation between delay time with size of data transmitted can be seen on the Figure 13 and in the formulation as below:

- Current TCP

$$
\mathrm{Y}=-9.10^{-12} \cdot \mathrm{X}^{3}+2 \cdot 10^{-7} \cdot \mathrm{X}^{2}+0.0004 \cdot \mathrm{X}+2.6975
$$

- IBR-DTN

$$
\begin{aligned}
\mathrm{Y}=4 \cdot 10^{-12} \cdot \mathrm{X}^{3}-2 \cdot 10^{-7} \cdot \mathrm{X}^{2}+0.0049 . \mathrm{X}+8.5978 \\
\mathrm{Y}=\text { Delay time }(\mathrm{s}), \\
\mathrm{X}=\text { Data Transmitted Size (Byte) }
\end{aligned}
$$

\subsubsection{Experiment 4 (Node on Mobile position, on LoS Condition)}

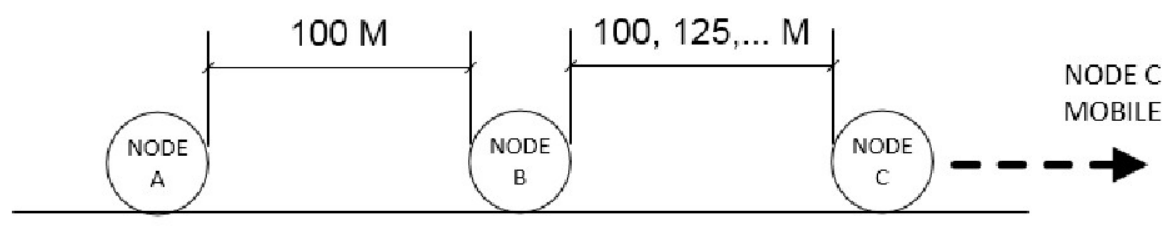

Figure 14. Node Position Experiment 4

Node A and Node B on the fix position while Node $C$ on mobile position. Node C sent 2000 bytes ICMP packet data size to Node A trough Node B to check the delay time and packet loss. In the throughput test Node A as iperf server and Node $\mathrm{C}$ as iperf client. 


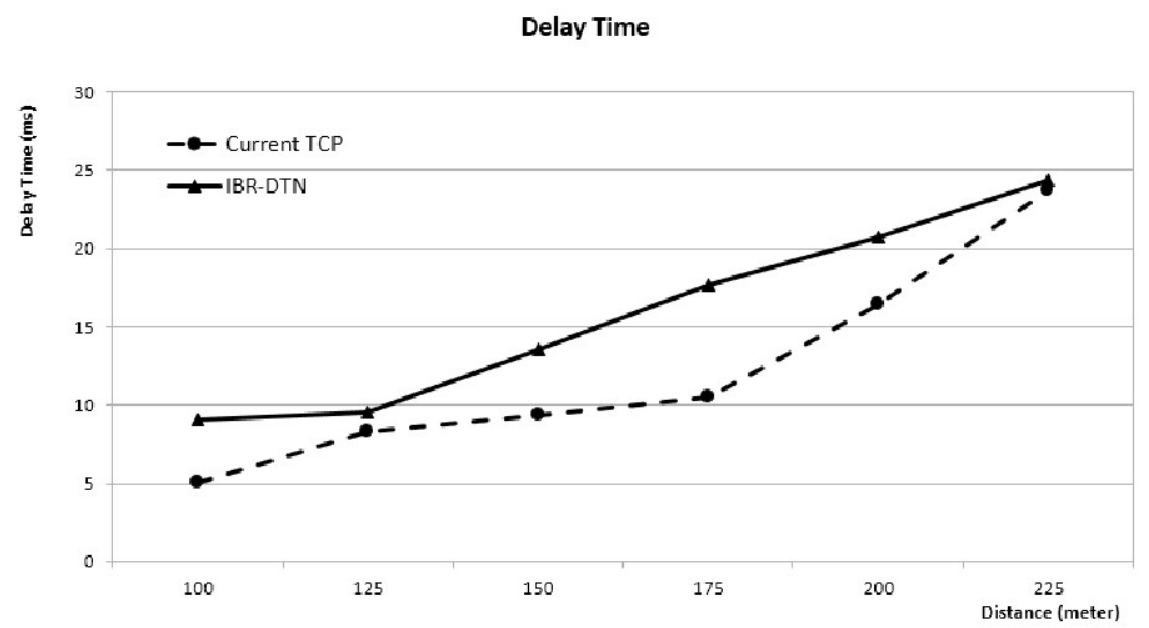

(a)

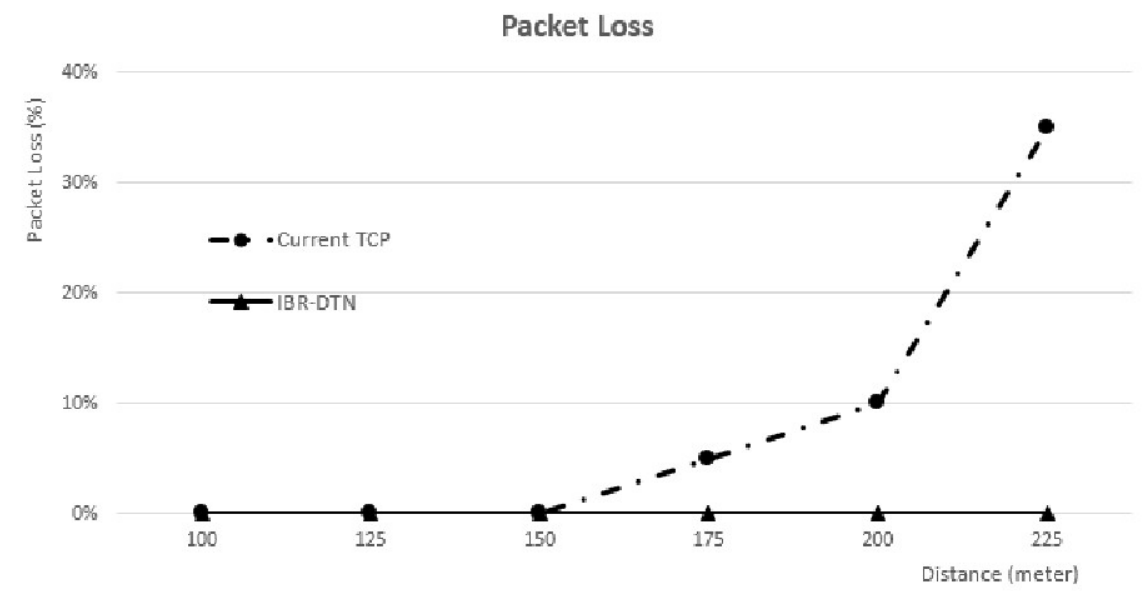

(b)

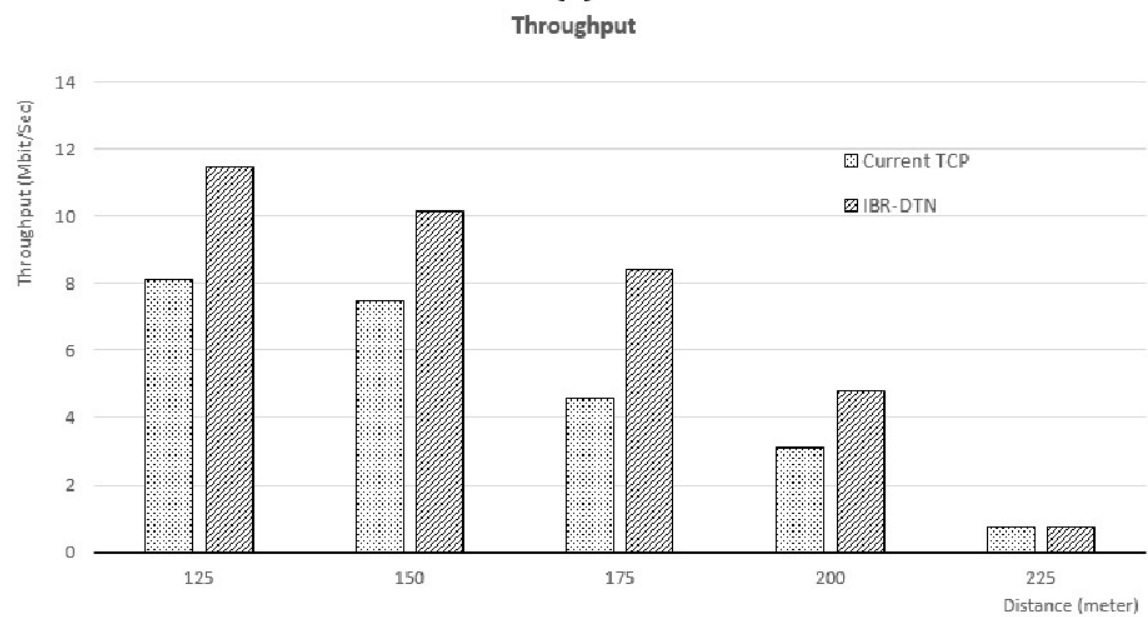

(c)

Figure 15. Packet Loss (a), Delay Time (b) and Throughput (c) (Mobile Position on LoS condition)

Based on Figure 15, we found that throughput wireless mesh with IBR-DTN higher than Current TCP on the LoS area with node on mobile position about 
28\%. The delay time measurement result was almost the same result between Current TCP and IBR-DTN but IBR-DTN could keep wireless mesh networks from packet loss by using store and forward mechanism.
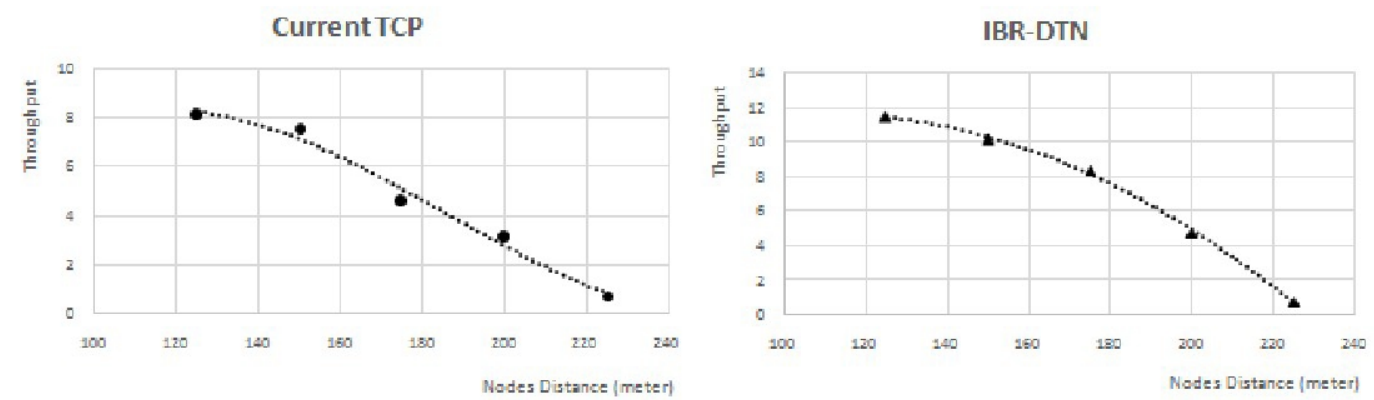

Figure 16. Throughput to Nodes Distance

(Mobile Position on LoS condition)

We also obtained the relation between throughput and nodes distance by using polynomial function and calculation.

- Current TCP

$$
Y=7 \cdot 10^{-6} \cdot X^{3}-0.0039 . X^{2}+0.6234 . X-22.972
$$

- IBR-DTN

$$
\mathrm{Y}=2.10^{-7} \cdot \mathrm{X}^{3}-0.001 . \mathrm{X}^{2}+0.205 . \mathrm{X}+0.2538
$$

Formula 9 and 10 indicate the relation of throughput with nodes distance. Y is throughput and $\mathrm{X}$ is nodes distance.

We calculated the Parson correlation coefficient to analyze the correlation delay time with packet data size and nodes distance.

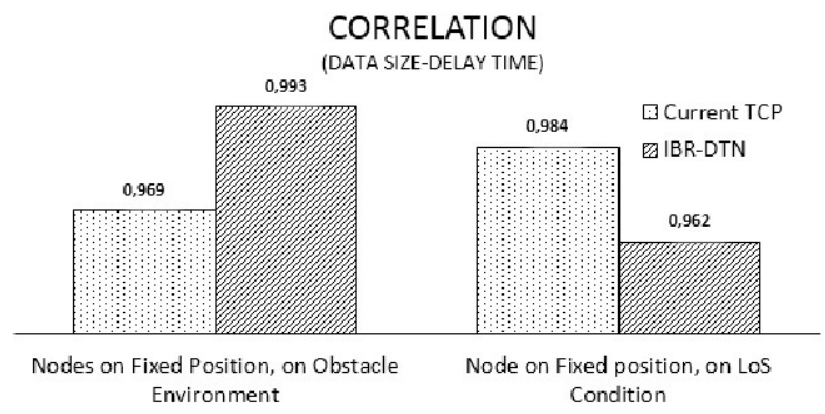

(a) 


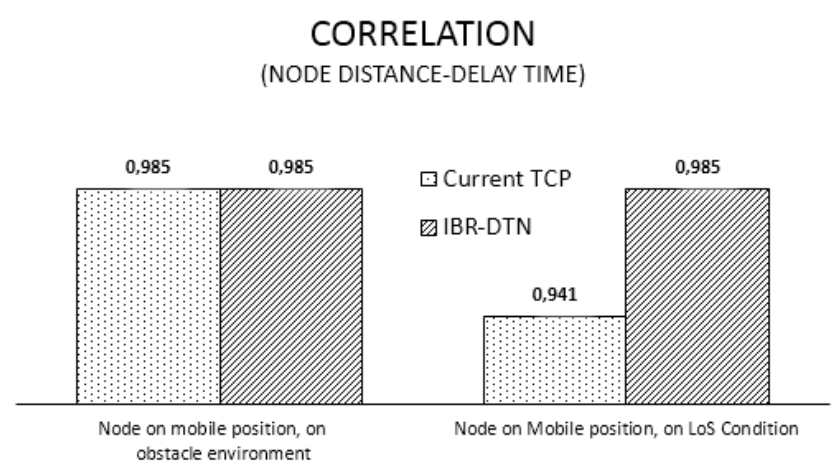

(b)

Figure 17. The Correlation Data Transmitted (a) and Nodes Distance to the Delay Time (b).

According to above data, the correlation of data transmitted and Delay Time was perfectly positive or very strong $[12,13]$ and the correlation of nodes distance to the delay time also very strong.

\section{CONCLUSION}

The widely reporting that IBR-DTN can work together with Batman-adv routing protocol. This paper proves that performance of IBR-DTN is very good on the wireless mesh networks with intermittent connectivity. Our experiments show that IBR-DTN implementation can reduce packet loss in the wireless mesh networks. Batman-adv also can be combined with IBRDTN on wireless mesh networks. The delay time of IBR-DTN in our experiment was higher than Current TCP although the packet size and node distance also influence to the delay time. The throughput performance of IBR-DTN was also higher than Current TCP wireless mesh networks (without DTN application) on the all of experiment. The best IBR-DTN performance was occur on the wireless mesh with LoS condition. In the our experiment research also shows that the size of packet data transmitted, the distance between nodes and the environmental conditions greatly affect to the value of the delay time, packet loss and throughput. It is important to continue this research especially with add another aspect to see performance IBR-DTN with different aspect and condition. We also want to continue this research with more complex nodes and embedded system application.

\section{Acknowledgements}

This research supported by ER2C Lab, DIKTI Funding Scholarship (BPPDN) and DIKTI PKLN 2015 (Penelitian Kerjasama Luar Negeri).

\section{REFERENCES}

1. Karisma Babu, Luis Miguel Cortes-Pena, Prateek Shah, Shivaranjani Sankara Krishnan, Wireless Mesh Network Implementation, School of Electrical Engineering Georgia Institute of Technology Atlanta, GA ,2007. 
2. Jeremiah Scholl, Lambros Lambrinos, Anders Lindgren, Rural Telemedicine Networks Using Store- and-Forward Voice-over-IP, Norwegian Centre for Telemedicine, University Hospital of North Norway , Ed 1, pp 448-452, 2009.

3. Tianle Zhang, Chunlu Wang, Zongwei Luo, Delay Tolerant Vehicular Network in Intelligent Transportation System, ; International Conference on Computer and Communications Security, Hongkong, pp. 36, 2009.

4. Herman Yuliandoko, Sritrusta Sukaridhoto, M Udin Harun Al Rasyid, Design of Implementation Delay Tolerant at Wireless Mesh Networks Using IBR-DTN and Batman-Adv, The ASEAN Conference on Science and Technology (COSAT) 2014, Bogor Indonesia, pp. 365-376, 2014.

5. Davinder Singh Sandhu, Sukesha Sharma, Performance evaluation of Batman, DSR, OLSR routing protocol; International Journal of Emerging Technology Advance Engineering (IJETAE), India, ISSN 22502459, Volume 2, pp. 184-188, 2012.

6. Daniel Seither, Andre Konig, Matthias Hollick, Routing Performance of Wireless Mesh Networks: A Practical Evaluation of BATMAN Advanced, IEEE 11th Conference on Local Computer Networks, BonnGermany, pp. 897-904, 2011.

7. Michael Doering, Sven Lahde, Johannes Morgenroth, and Lars Wolf, IBRDTN: An Efficient Implementation for Embedded Systems, Proceedings of the third ACM workshop on Challenged networks CHANTS '08, Braunschweig-Germany, pp. 117-119, 2008.

8. Laurent Delosieres, Simin Nadjm-Tehrani, BATMAN Store-andForward: the Best of Two Worlds, IEEE International Conference on Pervasive Computing and Communications Workshops, LinkopingSweden, pp. 727-733, 2012.

9. K. Scott, S. Burleigh. Bundle Protocol Specification. RFC 5050, The MITRE Corporation, NASA Jet Propulsion Laboratory, 2007.

10. Warthman Forest, Delay-Tolerant Networks (DTNs): A Tutorial v1.1, Warthman Assosiates, Ed. 2, pp 7-19, 2012.

11. Telecommunications and Internet Protocol Harmonization Over Networks (TIPHON), General aspects of Quality of Service (QoS), TR 101329 V2.1.1, 1999.

12. Kelly H. Zou, Kemal Tuncali, Stuart G. Silverman, Correlation an Simple Linier Regression, Boston-US, Ed.1, pp. 617-622, 2003.

13. Sugiyono, Statistika Untuk Penelitian, CV. Alfabeta Bandung Indonesia, Ed. 10, pp. 269-297, 2009. 